\title{
Incidencia del déficit cognitivo postoperatorio en anestesia regional y general en una institución de cuarto nivel en Bucaramanga (Santander, Colombia) en el año 2017
}

Postoperative cognitive dysfunction incidence for regional and general anesthesia in a level four institution in Bucaramanga (Santander, Colombia) during 2017

Incidência do déficit cognitivo pós-operatório em anestesia regional e geral em uma instituição de quarto nível em Bucaramanga (Santander, Colômbia) em 2017

Francisco Javier Contreras-Forero, MD. Esp. ${ }^{1}$ iD, Miguel Enrique Ochoa, MD. MSc. ${ }^{2}$ (D) Margarita Perez, MD. ${ }^{3}$ (iD, Mauricio Pinillos, MD. ${ }^{3}$ iD, Laura Celis, $M D^{3}$ (iD, Jhon Sebastián Valencia-Guampe, Est. ${ }^{4}$ (iD , Angi Yaquely Delgado-Pascuaza, Est. ${ }^{4}$ iD

1. Médico general, Universidad Industrial de Santander. Especialista en Anestesiología y Reanimación, Universidad Autónoma de Bucaramanga. Colombia.

2. Médico general, Magister en epidemiología, Universidad Industrial de Santander. Docente, Universidad Autónoma de Bucaramanga, Observatorio de Salud Pública de Santander. Colombia.

3. Médico general, Universidad Autónoma de Bucaramanga. Colombia.

4. Médico interno, Universidad del Cauca. Popayán. Colombia.

Correspondencia. Jhon Sebastián Valencia-Guampe. Universidad del Cauca. Río Blanco km2 Paraiso Real etapa 1 Popayán. Email.johnvalencia@unicauca.edu.co

\section{INFORMACIÓN DEL ARTÍCULO:}

Artículo recibido: 12 de septiembre de 2019

Artículo aceptado: 24 de septiembre de 2020

DOI: https://doi.org/10.29375/01237047.3711

Cómo citar: Contreras-Forero FJ, Ochoa ME, Perez M, Pinillos M, Celis L, Valencia-Guampe JS, et al. Incidencia del déficit cognitivo postoperatorio en anestesia regional y general en una institución de cuarto nivel en bucaramanga (santander, colombia) en el año 2017. MedUNAB. 2020;23(3): 450-463. Doi: https://doi.org/10.29375/01237047.3711

\section{RESUMEN}

Introducción. En el proceso de envejecimiento, la mayoría de los sistemas orgánicos mantienen su funcionamiento basal, pero existe una reducción de la reserva funcional 


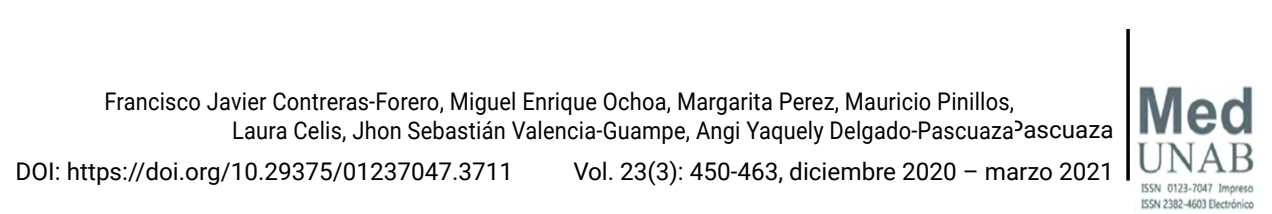

y de la capacidad para compensar el estrés fisiológico, lo que hace que los pacientes de mayor edad sean más propensos a complicaciones postoperatorias. Se realizó un estudio en instituciones de Bucaramanga para determinar la incidencia de déficit cognitivo postoperatorio y los factores de riesgo asociados. Metodología. Estudio observacional analítico de cohorte prospectivo realizado en instituciones de salud de Bucaramanga durante 2017 con pacientes mayores de 55 años intervenidos bajo anestesia regional, general balanceada o anestesia total intravenosa. Resultados. Se estudiaron 173 pacientes, 112 mujeres (64.74\%) y 61 hombres (35.26\%) operados. Las técnicas anestésicas más empleadas fueron: Regional (46.24\%) y General balanceada (44.51\%). La incidencia de Déficit Cognitivo Postoperatorio a corto plazo fue 6.36\% en pacientes previamente sanos, con valor P significativo (0.001) ajustado. Se detectó deterioro cognitivo del $51.41 \%$ con alteraciones en los dominios de lenguaje, atención y memoria; con aumento en la evaluación a largo plazo. Durante el seguimiento de pacientes a largo plazo resultó en un $11.55 \%$, determinando la incidencia de Déficit Cognitivo Postoperatorio tardío en un $17.91 \%$. La técnica anestésica con mayor proporción de déficit a largo plazo fue la anestesia mixta, comparada con anestesia regional. Discusión. En la actualidad no existe una definición estandarizada de Déficit Cognitivo Postoperatorio, se ha observado mayor frecuencia y mayor prolongación en pacientes de edad avanzada. El estudio demostró una incidencia temprana significativamente menor, comparándolo con artículos internacionales, pero una incidencia mayor en déficit postoperatorio tardío, resultado de mayores complicaciones intraoperatorias, comparado con otros estudios. Conclusiones. El Déficit Cognitivo Postoperatorio involucra múltiples factores de riesgo; en el estudio se encontró mayor asociación con la edad, la escolaridad, la hipotensión y la anestesia general. No hubo asociación entre comorbilidades y el Déficit Cognitivo Postoperatorio. Se determinó la presencia de hipotensión para desarrollo de este déficit.

Palabras claves:

Déficit Cognitivo Postoperatorio; Disfunción cognitiva; Incidencia; Anestesia intravenosa; Anestesia general; Anestesia balanceada.

\section{ABSTRACT}

Introduction. Within the aging process, most organic systems maintain their basal functions. However, there is a reduction in functional reserve and the capacity to compensate physiological stress, which makes older patients more prone to postoperative complications. A study was performed in institutions in Bucaramanga to determine the incidence of postoperative cognitive dysfunction and the associated risk factors. Methodology. This was a analytical observational prospective cohort study performed in healthcare institutions in Bucaramanga in 2017 with patients older than 55 years of age under regional, balanced general or total intravenous anesthesia. Results. One hundred and seventy-three operated patients were studied, among which 112 (64.74\%) were women and $61(35.26 \%)$ were men. The most commonly used anesthetic techniques were: regional (46.24\%) and balanced general (44.51\%). Short-term Postoperative Cognitive Dysfunction incidence was $6.36 \%$ in previously healthy patients, with an adjusted significant $P$ value $(0.001)$. Cognitive deterioration was detected in $51.41 \%$ of patients, with alterations in their mastery of language, attention and memory. This increased for the long-term evaluation. During long-term patient follow-up, it resulted in $11.55 \%$, determining the incidence of delayed Postoperative Cognitive Dysfunction at $17.91 \%$. The anesthetic technique with the highest proportion of longterm dysfunction was mixed anesthesia, compared to regional anesthesia. Discussion. There is currently no standardized definition for Postoperative Cognitive Dysfunction. It has been observed more frequently and for longer extensions in older patients. The study demonstrated a significantly lower early incidence when compared to international articles, but a greater incidence of delayed postoperative dysfunction as a result of more intraoperative complications compared to other studies. Conclusions. Postoperative Cognitive Dysfunction involves multiple risk factors. The study observed a greater association with age, level of education, hypotension and general anesthesia. There was no association between comorbidities and Postoperative Cognitive Dysfunction. The presence of hypotension was determined for developing this dysfunction.

\section{Keywords:}

Postoperative Cognitive Dysfunction; Cognitive dysfunction; Incidence; Intravenous anesthesia; General anesthesia; Balanced anesthesia. 


\section{RESUMO}

Introdução. No processo de envelhecimento, a maioria dos sistemas orgânicos mantém seu funcionamento basal, mas há uma diminuição da reserva funcional e da capacidade de compensar o estresse fisiológico que torna os pacientes idosos mais sujeitos a complicações pós-operatórias. Foi realizado um estudo em instituições da cidade de Bucaramanga para determinar a incidência de déficit cognitivo pós-operatório e os fatores de risco associados. Métodos. Estudo observacional analítico de coorte prospectivo realizado em instituições de saúde em Bucaramanga durante o ano de 2017 em pacientes com idade superior a 65 anos operados sob anestesia regional, geral balanceada ou anestesia geral intravenosa. Resultados. Foram estudados 173 pacientes, 112 mulheres (64.74\%) e 61 homens (35.26\%) operados. As técnicas anestésicas mais utilizadas foram: regional (46.24\%) e geral balanceada (44.51\%). A incidência de déficit cognitivo pós-operatório de curto prazo foi de $6.36 \%$ em pacientes previamente saudáveis, com um valor P significativo (0.001) ajustado. Detectouse deterioração cognitiva de 51.41\% com alterações nos domínios da linguagem, atenção e memória; com aumento na avaliação de longo prazo. No seguimento a longo prazo dos pacientes resultou em $11.55 \%$, determinando a incidência de déficit cognitivo pós-operatório tardio em 17.91\%. A técnica anestésica com maior proporção de déficit no longo prazo foi a anestesia mista, em comparação com a anestesia regional. Discussão. Atualmente não existe uma definição padronizada de Déficit Cognitivo Pós-operatório, a qual observa-se com maior frequência e por mais tempo em pacientes idosos. O estudo demonstrou incidência precoce significativamente menor, em comparação com resultados de artigos internacionais, mas maior incidência de déficit pós-operatório tardio, resultado de maiores complicações intraoperatórias, em comparação com outros estudos. Conclusões. O Déficit Cognitivo Pós-operatório envolve múltiplos fatores de risco; o estudo encontrou maior associação com idade, escolaridade, hipotensão e anestesia geral. Não houve associação entre comorbidades e Déficit Cognitivo Pós-operatório. Determinou-se a presença de hipotensão para o desenvolvimento desse déficit.

Palavras-chave:

Déficit Cognitivo Pós-operatório; Disfunção cognitiva; Incidência; Anestesia intravenosa; Anestesia geral; Anestesia balanceada.

\section{Introducción}

En el proceso de envejecimiento, la mayoría de los sistemas orgánicos mantienen su funcionamiento basal aunque existe una reducción de la reserva funcional y de la capacidad para compensar el estrés fisiológico. Por esto se presenta un aumento en las complicaciones postoperatorias a mayor edad de los pacientes. Probablemente, una de las más habituales es la aparición de Déficit Cognitivo Postoperatorio (DCPO), definido como una alteración cognitiva nueva que aparece después de un procedimiento quirúrgico. A diferencia del delirium postoperatorio, no compromete la conciencia y se caracteriza por ser un cuadro sutil, difícil de detectar que afecta la atención, la memoria, el aprendizaje, la visión espacial, las habilidades motoras, la ejecución y puede acompañarse de cambios de comportamiento (1). Esta entidad está formada por un espectro variable de trastornos neurológicos, que pueden aparecer con una incidencia variable de entre el 5\% y el $50 \%$ de los ancianos, y que oscila desde el delirio postoperatorio leve y transitorio hasta el desarrollo de un trastorno cognitivo permanente (2).

El tiempo de aparición de DCPO se caracteriza en su primera etapa por una incidencia bimodal. En la primera semana del postquirúrgico (7 a 8 días) durante la que se presenta un compromiso transitorio a corto plazo; ocurre un declive en la capacidad cognitiva. Este descenso inicial permite predecir un deterioro cognitivo asociado con demencia de 3 a 5 años después de la cirugía, con tasas de hasta $70 \%$ en los pacientes que tienen 65 años o más (3). El DCPO temprano ocurre en pacientes quirúrgicos adultos de todas las edades, pero los pacientes ancianos presentan mayor riesgo

El DCPO tardío empieza 3 meses después de la cirugía. Este deterioro cognitivo es todavía detectable en pacientes mayores de 60 años (4) con implicaciones potenciales a largo plazo para los pacientes, como el riesgo de dejar el trabajo prematuramente, o el deterioro general de la calidad de vida $(5,6)$. También se ha observado que el déficit constituye un aumento del riesgo de mortalidad $(7,8)$.

Diversos estudios ponen en duda la existencia del DCPO como entidad clínica, considerando que existe una remisión del cuadro en la mayoría de pacientes, lo que podría explicarse por la ocurrencia de dos escenarios diferentes de acuerdo al estado cognitivo previo del paciente: (a) en pacientes normales en los que la anestesia y la cirugía les producen una disfunción 
cognitiva temporal pero que logran una remisión completa, y (b) en pacientes con deterioro cognitivo leve anterior a la cirugía (y por tanto a la anestesia) a la que se suma la disfunción cognitiva usual en este tipo de procedimientos, haciendo más evidentes los problemas anteriores provocando la evolución de la condición preexistente.

Anivel nacional se cuenta con dos estudios. Uno de estos en pacientes mayores de 18 años de edad, militares que ingresan con trauma ortopédico y que fueron llevados a dos o más intervenciones quirúrgicas bajo anestesia en el Hospital Militar Central en Bogotá obteniéndose una incidencia de DCPO leve con $16.6 \%$ cuando se aplicó el MMSE (Mini-Mental State Examination) y de $6.6 \%$ cuando se aplicó el test de MOCA (Montreal cognitive assessment); en todos los casos se trató de Déficit Cognitivo Leve es decir ningún paciente obtuvo puntajes para déficit cognitivo moderado o severo. El otro estudio de Guzmán et al. (9) realizado en la ciudad de Bucaramanga en el Hospital Universitario de Santander a pacientes programados para cirugía en una población mayor de 50 años, utilizando como herramienta el MMSE. Se determinó que la incidencia de DCPO fue de $3.42 \%$. Este estudio se realizó porque en el país no se cuenta con suficiente literatura acerca del grado de deterioro cognitivo relacionado con la técnica anestésica utilizada. El objetivo fue evaluar el estado cognitivo del paciente post-anestésico después de una intervención quirúrgica y así determinar la incidencia de DCPO y los factores asociados en individuos mayores de 55 años.

\section{Metodología}

Durante el año 2017 se realizó un estudio observacional analítico de cohorte prospectivo en pacientes mayores de 55 años sometidos a intervenciones quirúrgicas con requerimiento de anestesia regional, general, mixta o anestesia total intravenosa (TIVA) en la Fundación Oftalmológica de Santander-Clínica Carlos Ardila Lulle (FOSCAL), junto con la Clínica FOSCAL internacional, ambas instituciones prestadoras de salud con servicios de alta complejidad y con influencia en el nororiente colombiano. Se realizó un muestreo no probabilístico por conveniencia. Para el cálculo del tamaño muestral, con el objetivo de detectar una incidencia de $12 \%$ y utilizando un poder del $80 \%$ con nivel de confianza del $95 \%$ y significancia del 3\%, el tamaño de muestra requerido fue de 222 pacientes, con ajuste de $10 \%$ por pérdidas.
Se aplicaron como criterios de inclusión pacientes que fueron programados para cirugía en la consulta ambulatoria, y que asistieron a la consulta externa de anestesiología para la valoración preanestésica. Estos serían sometidos a cirugía bajo diferentes técnicas anestésicas como anestesia regional, general balanceada o anestesia total intravenosa. Se excluyeron pacientes que requirieron ingreso en Unidad de cuidados intensivos (UCI) durante su hospitalización, pacientes con analfabetismo, con trastornos neurológicos de base o patologías psiquiátricas, con deterioro cognitivo previo, también a pacientes sometidos a más de una cirugía bajo anestesia posterior al ingreso en el estudio, y con historial de consumo de sustancias psicoactivas, así como pacientes sometidos a neurocirugía (excepto de columna).

A los pacientes que cumplían con los criterios se les registraron los datos sociodemográficos con las variables: edad, sexo, peso, talla, índice de masa corporal (IMC, tipo de anestesia, tiempo de anestesia, escolaridad, ASA, clase funcional definida por la actividad física del paciente, medicamentos, diagnóstico prequirúrgico, antecedentes patológicos y monitoria completa. Se empleó un análisis estadístico multivariado para determinar los diferentes factores de riesgo de DCOP.

Previa firma del consentimiento informado se aplicó como instrumento la escala de evaluación el MMSE (Figura 1), que es una prueba neuropsicológica desarrollada por Folstein et al. en 1975 como un instrumento práctico para la evaluación del estado cognitivo, el cual ha sido extensamente utilizado tanto en la práctica clínica como en el campo de la investigación $(10,11)$. El MMSE es una prueba que consta de ciertas tareas que se le piden al paciente para evaluar una serie de 11 dominios:

1. Orientación temporal

2. Orientación espacial

3. Recuerdo inmediato de 3 palabras

4. Atención o cálculo (deletreo en inversa o resta secuencial)

5. Recuerdo diferido de las tres palabras

6. Nominación de 2 objetos

7. Repetición de una frase

8. Comprensión de una orden verbal

9. Comprensión de una orden escrita

10.Escritura de una oración

11. Copia de un diagrama 
Figura 1. Puntaje de Mini-mental State Examination estratificado por edad y escolaridad.

\section{MINI MENTAL STATE EXAMINATION}

(MMSE)

Basado en Folstein et al. (1975), Lobo et al. (1979)

Nombre:

Fecha:

Estudios/Profesión:

Observaciones:
F. nacimiento:

N. $\mathrm{H}^{\mathrm{a}}$ :

\section{Varón [ ] Mujer [ ] Edad:}

\begin{tabular}{|c|c|c|c|}
\hline \multicolumn{3}{|c|}{$\begin{array}{ll}\text { ¿En qué año estamos? } & 0-1 \\
\text { ¿En qué estación? 0-1 } & \\
\text { ¿En qué día (fecha)? } & 0-1 \\
\text { ¿En qué mes? } & 0-1 \\
\text { ¿En qué día de la semana? } & 0-1\end{array}$} & $\begin{array}{l}\text { ORIENTACIÓN } \\
\text { TEMPORAL (Máx.5) }\end{array}$ \\
\hline \multicolumn{2}{|c|}{$\begin{array}{l}\text { ¿En qué hospital (o lugar) estamos? } \\
\text { ¿En qué piso (o planta, sala, servicio)? } \\
\text { ¿En qué pueblo (ciudad)? } \\
\text { ¿En qué provincia estamos? } \\
\text { ¿En qué país (o nación, autonomía)? }\end{array}$} & $\begin{array}{l}0-1 \\
0-1 \\
0-1 \\
0-1 \\
0-1\end{array}$ & $\begin{array}{l}\text { ORIENTACIÓN } \\
\text { ESPACIAL (Máx.5) }\end{array}$ \\
\hline \multicolumn{3}{|c|}{ 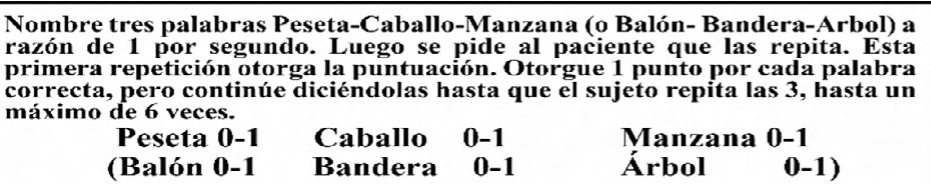 } & $\begin{array}{l}\mathrm{N}^{\circ} \text { de repeticiones } \\
\text { necesarias } \\
\text { FIJACIÓN-Recuerdo } \\
\text { Inmediato (Máx.3) }\end{array}$ \\
\hline \multicolumn{3}{|c|}{$\begin{array}{l}\text { Si tiene } 30 \text { pesetas y me va dando de tres en tres, ¿Cuántas le van quedando?. } \\
\text { Detenga la prueba tras } 5 \text { sustraciones. Si el sujeto no puede realizar esta } \\
\text { prueba, pídale que deletree la palabra MUNDO al revés. }\end{array}$} & \multirow{2}{*}{$\begin{array}{l}\text { ATENCIÓN- } \\
\text { CÁLCULO (Máx.5) }\end{array}$} \\
\hline $\begin{array}{ll}300-1 & 270-1 \\
\left(O_{0-1}\right. & \text { D 0-1 }\end{array}$ & $\begin{array}{l}240-1 \\
\mathrm{~N}_{0-1}\end{array}$ & $\begin{array}{ll}210-1 & 180-1 \\
\text { U } 0-1 & \text { Mo-1) } \\
\end{array}$ & \\
\hline \multicolumn{3}{|c|}{$\begin{array}{l}\text { Preguntar por las tres palabras mencionadas anteriormente. } \\
\begin{array}{rlll}\text { Peseta 0-1 } & \text { Caballo } 0-1 & \text { Manzana 0-1 } \\
\text { (Balón 0-1 } & \text { Bandera } 0-1 & \text { Árbol } & 0-1 \text { ) }\end{array}\end{array}$} & $\begin{array}{l}\text { RECUERDO diferido } \\
\text { (Máx.3) }\end{array}$ \\
\hline \multicolumn{3}{|c|}{$\begin{array}{l}\text { DENOMINACIÓN. Mostrarle un lápiz o un boligrafo y preguntar zqué es } \\
\text { esto?. Hacer lo mismo con un reloj de pulsera. Lápiz 0-1 Reloj 0-1 } \\
\text {.REPETICIÓN. Pedirle que repita la frase: "ni sí, ni no, ni pero" (o "En } \\
\text { un trigal había } 5 \text { perros") 0-1 } \\
\text { ORDENES. Pedirle que siga la orden: "coja un papel con la mano derecha, } \\
\text { dóblelo por la mitad, y póngalo en el suelo". } \\
\text { Coje Con mano d. 0-1 dobla por mitad 0-1 pone en suelo 0-1 } \\
\text { LECTURA. Escriba legiblemente en un papel "Cierre los ojos". Pídale que lo } \\
\text { lea y haga lo que dice la frase 0-1 } \\
\text {.ESCRITURA. Que escriba una frase (con sujeto y predicado) 0-1 } \\
\text {.COPIA. Dibuje } 2 \text { pentágonos intersectados y pida al sujeto que los copie tal } \\
\text { cual. Para otorgar un punto deben estar presentes los } 10 \text { ángulos y la } \\
\text { intersección. 0-1 }\end{array}$} & LENGUAJE (Máx.9) \\
\hline Puntuaciones de referencia & $\begin{array}{l}27 \text { ó más: normal } \\
24 \text { ó menos: sospecha } p\end{array}$ & $\begin{array}{l}\text { ológica } \\
\text { 12-24: deterioro } \\
\text { 9-12: demencia }\end{array}$ & $\begin{array}{l}\text { Puntuación Total } \\
\text { (Máx.: } 30 \text { puntos) }\end{array}$ \\
\hline
\end{tabular}

Fuente: tomado de Rev. Neurol. Arg. 26 (1) 11:15,200137

Los puntajes parciales son sumados obteniendo un máximo de 30 puntos, clasificando al individuo en una escala de función cognitiva, utilizando una puntuación de corte de 23. Para la detección de delirio o demencia en pacientes hospitalizados, la sensibilidad y especificidad del MMSE son de $87 \%$ y $82 \%$, respectivamente. Sin embargo, aunque es una prueba de detección de problemas, no identifica trastornos específicos (12). Se programaron 3 momentos para la realización del
MMSE: previo a la cirugía, 7 días posterior a el evento quirúrgico y, por último, después de 3 meses.

El proyecto fue presentado al Comité de Postgrado de la Universidad Autónoma de Bucaramanga. Luego de la aprobación de este comité fue presentado al Comité de Etica de la FOSCAL. El 16 de diciembre de 2016 se aprobó también por esta entidad, por lo que se sostiene que el estudio ostenta cierto rigor de calidad 
técnica y científica, y además cumple con los aspectos reglamentario y éticos planteados por el CEI-FOSCAL.

\section{Resultados}

Durante un periodo de 8 meses se tuvieron en cuenta 780 pacientes de los cuales 222 cumplían con los criterios de inclusión. Fueron evaluados individualmente por un periodo de 3 meses. Se presentaron 41 renuncias voluntarias de pacientes y la muerte de un paciente. 7 de los pacientes estudiados tenían deterioro cognitivo de base; sus datos se retiraron y no se incluyeron dentro del estudio. Así, se hizo seguimiento a un total de 173 pacientes. (Tabla 1 y Figura 2).

Tabla 1. Características generales y datos sociodemográficos de los pacientes.

\begin{tabular}{|c|c|c|}
\hline Mujer & 112 & $64.74 \%$ \\
\hline Hombre & 61 & $35.26 \%$ \\
\hline \multicolumn{3}{|l|}{ Grupos de edad (años) } \\
\hline 50 a 59 & 52 & $30.06 \%$ \\
\hline 60 a 64 & 35 & $20.23 \%$ \\
\hline 65 a 69 & 29 & $17.66 \%$ \\
\hline 70 a 74 & 21 & $12.14 \%$ \\
\hline 75 o más & 36 & $20.81 \%$ \\
\hline \multicolumn{3}{|l|}{ Escolaridad } \\
\hline Postgrado & 16 & $9.25 \%$ \\
\hline Pregrado & 24 & $13.87 \%$ \\
\hline Bachiller & 70 & $40.46 \%$ \\
\hline Primaria & 54 & $31.21 \%$ \\
\hline Primaria incompleta & 9 & $5.20 \%$ \\
\hline \multicolumn{3}{|l|}{ Procedencia } \\
\hline Urbana & 222 & $100 \%$ \\
\hline Otra & 5 & $2.89 \%$ \\
\hline \multicolumn{3}{|l|}{ Especialidad que intervenía quirúrgicamente } \\
\hline Cirugía vascular periférica & 9 & $5.2 \%$ \\
\hline ORL & 8 & $4.62 \%$ \\
\hline Cirugía de tórax & 1 & $0.85 \%$ \\
\hline Cirugía de cabeza y cuello & 5 & $2.89 \%$ \\
\hline Cirugía plástica & 3 & $1.73 \%$ \\
\hline Ginecología & 12 & $6.94 \%$ \\
\hline Cirugía general & 43 & $24.86 \%$ \\
\hline Urología & 27 & $15.61 \%$ \\
\hline Cirugía oncológica & 7 & $4.05 \%$ \\
\hline
\end{tabular}

Fuente: Elaboración propia 
Figura 2. Pacientes incluidos en el estudio.

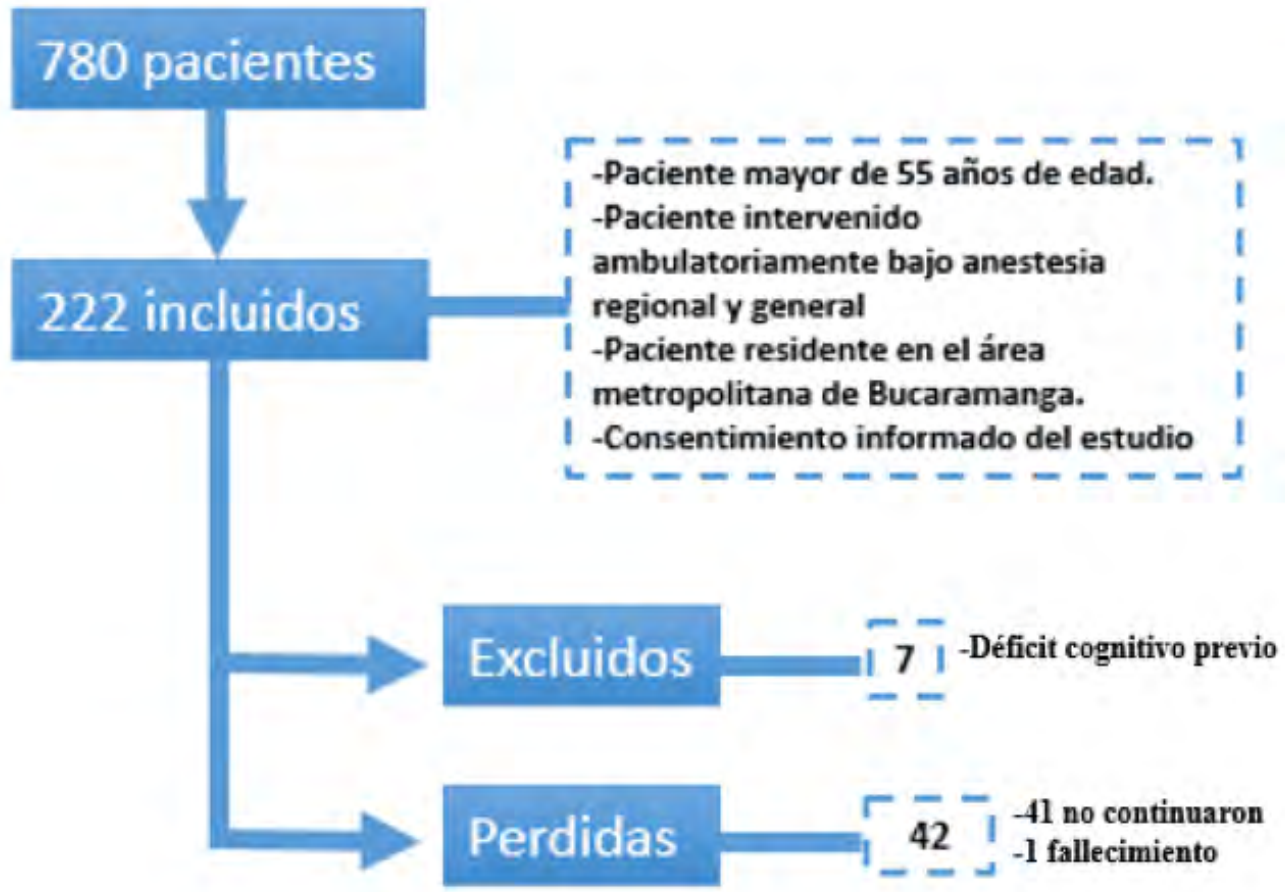

Fuente: elaborado por los autores

En la valoración preanestésica se tuvo en cuenta la clasificación de la American Society of Anesthesiologists (ASA) y La Clasificación Funcional de la New York Heart Association, en las que se evidenció el predominio de pacientes con bajo riesgo anestésico y pacientes con clase funcional I.

Las técnicas anestésicas más utilizadas fueron la Regional (46.24\%) y General balanceada (44.51\%)
(Figura 3). En cuanto a los eventos intraoperatorios y traslados a UCI se reportó hipotensión considerada con cifras de tensión arterial menor a 90/60 $\mathrm{mmHg}$. En mayor medida, con un $9.25 \%(n=16)$, un total de 15 pacientes requirió uso de vasopresor por hipotensión sostenida, y por el contrario solo se presentó un evento de desaturación y un traslado a UCI (Tabla 2).

Figura 3. Técnicas anestésicas empleadas.

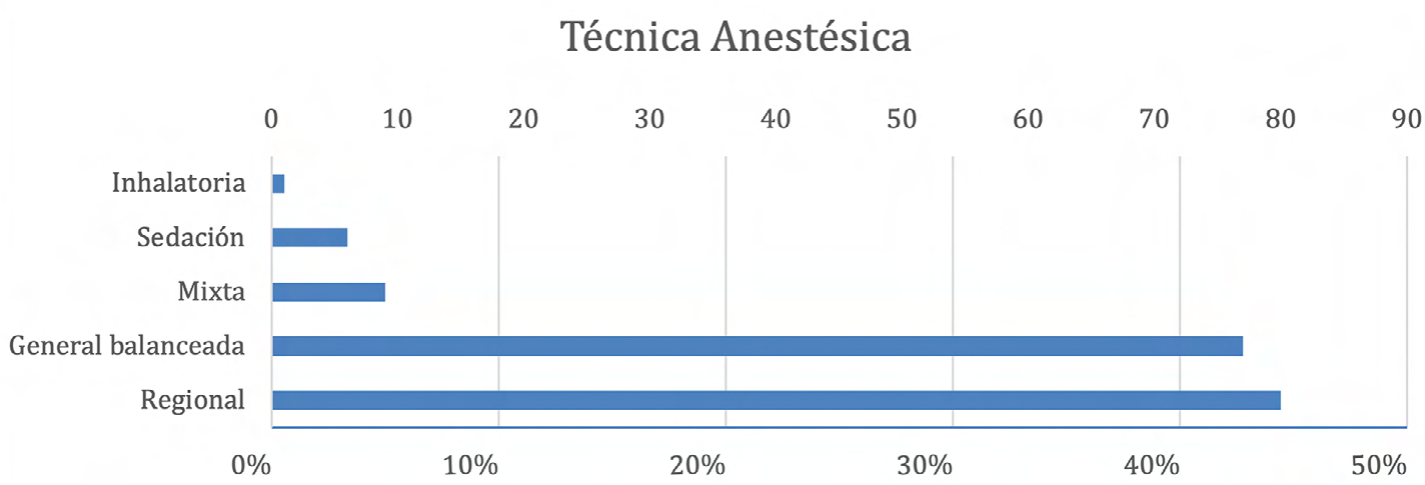

Fuente: elaborado por los autores 
Tabla 2. Eventos intraoperatorios.

\begin{tabular}{|c|c|c|}
\hline & Frecuencia & Porcentaje \\
\hline \multicolumn{3}{|l|}{ HIPOTENSIÓN } \\
\hline No & 157 & $90.75 \%$ \\
\hline Sí & 16 & $9.25 \%$ \\
\hline Total & 173 & $100.00 \%$ \\
\hline \multicolumn{3}{|c|}{ USO DE VASOPRESOR } \\
\hline No & 158 & $91.33 \%$ \\
\hline Sí & 15 & $8.61 \%$ \\
\hline Total & 173 & $100.00 \%$ \\
\hline \multicolumn{3}{|c|}{ DESATURACIÓN } \\
\hline No & 172 & $99.42 \%$ \\
\hline Sí & 1 & $0.58 \%$ \\
\hline Total & 173 & $100.00 \%$ \\
\hline \multicolumn{3}{|l|}{ UCI } \\
\hline No & 172 & $99.42 \%$ \\
\hline Sí & 1 & $0.58 \%$ \\
\hline Total & 173 & $100.00 \%$ \\
\hline \multicolumn{3}{|l|}{ UCI } \\
\hline No & 172 & $99.42 \%$ \\
\hline Sí & 1 & $0.58 \%$ \\
\hline Total & 173 & $100.00 \%$ \\
\hline
\end{tabular}

Fuente: elaborado por los autores

\section{Incidencia de déficit cognitivo postoperatorio a corto plazo}

El rango del puntaje en la prueba MMSE en preoperatorio, a corto y a largo plazo estuvo entre 26 y 30 , y los puntajes tuvieron una distribución asimétrica. Al comparar los cambios en el puntaje de la MMSE se encontró una disminución de la mediana de un punto y el puntaje más bajo se presentó en el tercer seguimiento con 26 puntos en comparación con el primer evento. Todos los cambios en la escala global no superaron los 2 puntos por encima o por debajo del puntaje inicial. La incidencia de déficit cognitivo postoperatorio a corto plazo fue de $6.36 \%(n=11)$ en los pacientes previamente sanos, con valor $\mathrm{P}$ significativo (0.001) y ajustado por edad y escolaridad. Se presentó deterioro cognitivo significativo del $51.41 \%$, con mayores alteraciones en los dominios de lenguaje, atención y memoria, y aumentando a largo plazo en comparación con la evaluación a corto plazo estadísticamente significativo (Tablas 3 y 4 ).

Tabla 3. Incidencia de déficit cognitivo a corto y largo plazo.

$\begin{array}{ccccc}\text { Deterioro cognitivo } & \text { Casos (\%) } & \text { A corto plazo (7 días) } & \text { A largo plazo (90 días) } & \text { P } \\ & & 69(39.88 \%) & 89(51.41 \%) & <0.02 \\ \text { Déficit cognitivo } & \text { Casos (\%) } & 11(6.36 \%) & 31(17.92 \%) & <0.001\end{array}$

Fuente: elaborado por los autores 
Tabla 4. Deterioro cognitivo por dominios a corto y largo plazo

\begin{tabular}{lr} 
Dominios & A cort \\
\hline Orientación & 2 \\
\hline Repetición & 37 \\
\hline Atención & 37 \\
Memoria & 46 \\
\hline Lenguaje & \\
borado por los autores & \\
\hline & \\
eia de déficit cognitivo &
\end{tabular}

Durante el seguimiento posterior de los pacientes a largo plazo para determinar el déficit cognitivo tardío se encontró en $11.55 \%$ la incidencia de déficit, con una incidencia total de $17.91 \%$ para DCPO tardío (90 días). En cuanto a las técnicas anestésicas, se presentó una mayor proporción de casos con déficit a largo plazo con anestesia mixta, con un RR de 2.75 (IC 1.53-5) y una diferencia significativamente estadística ( $\mathrm{p}$ 0.001) comparada con la técnica regional, en la que no se afecta el estado de conciencia.
De los eventos intraoperatorios, la hipotensión con un RR de 2.86 (IC 1.46-5.57) y una diferencia significativamente estadística ( $\mathrm{p}$ 0.002) en comparación con los pacientes que mantienen una tensión arterial estable durante la cirugía. Adicionalmente se evidenció que con cada década por encima de los 55 años el riesgo de presentar déficit cognitivo aumenta con un RR de 1.03 (IC 1.041.05) y una diferencia significativamente estadística ( $\mathrm{p}$ 0.001 ) con respecto a la población menor de esta edad. La última variable estadísticamente significativa fue el sexo, pues donde se encontró que, en la población de sexo masculino, un RR de 1.8 (IC 1.44-2.24) y una diferencia significativamente estadística (p 0.001) a la del sexo femenino. Las demás variables no presentaron hallazgos representativos en el estudio (Tabla 5).

Tabla 5. Deterioro cognitivo por dominios a corto y largo plazo

\section{Incidencia \\ DCPO**}

\begin{abstract}
RR CRUDO (IC95\%)
\end{abstract}

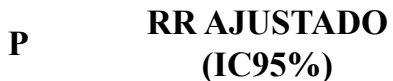

\section{FACTOR DE RIESGO}

ANESTESIA

\begin{tabular}{|cccccc}
\hline Regional $(\mathrm{N}=80)$ & $12(15 \%)$ & Referencia & Referencia & \\
\hline General balanceada $(\mathrm{N}=77)$ & $15(19.48 \%)$ & $1.3(0.65-2.6)$ & 0.74 & $1.52(0.72-3.27)$ & 0.27 \\
\hline Sedación $(\mathrm{N}=6)$ & $1(16.67 \%)$ & $1.11(0.17-7.16)$ & 0.91 & $3.16(0.63-16.3)$ & 0.17 \\
\hline Mixta $(\mathrm{N}=9)$ & $3(33.33 \%)$ & $2.22(0.77-6.42)$ & 0.14 & $2.75(1.53-5)$ & 0.001 \\
\hline Inhalatoria $(\mathrm{N}=1)$ & $0(0 \%)$ & & - & & - \\
\hline ESCOLARIDAD & & & & Referencia & \\
Primaria incompleta $(\mathrm{N}=9)$ & $1(11.11 \%)$ & Referencia & & $2.92(0.41-21.15)$ & 0,29 \\
Primaria $(\mathrm{N}=54)$ & $11(20.37 \%)$ & $1.83(0.27-12.52)$ & 0.54 & 0,1 \\
\hline bachillerato $(\mathrm{N}=70)$ & $17(24.29 \%)$ & $2.19(0.33-14.52)$ & 0.49 & $5.13(0.72-36.72)$ & 0,44 \\
\hline Pregrado $(\mathrm{N}=24)$ & $2(8.33 \%)$ & $0.75(0.77-7.3)$ & 0.8 & $2.54(0.24-26.78)$ & 0,44 \\
Postgrado $(\mathrm{N}=16)$ & $0(0 \%)$ & & & & Referencia \\
No Hipotensión $(\mathrm{N}=157)$ & $24(15.29 \%)$ & Referencia & & $3.66(2-6.67)$ & $<0,001$ \\
\hline Hipotensión $(\mathrm{N}=16)$ & $7(43.75)$ & $2.86(1.46-5.57)$ & 0.002 & & \\
\hline
\end{tabular}




\begin{tabular}{cccccc} 
Edad (Continua) & - & $1.05(1.01-1.08)$ & 0.003 & $1.04(1.03-1.05)$ & $<0,001$ \\
Sexo Femenino $(\mathrm{N}=112)$ & $18(16.07 \%)$ & Referencia & \multicolumn{3}{c}{ Referencia } \\
Sexo Masculino $(\mathrm{N}=61)$ & $13(21.31 \%)$ & $1.33(0.7-2.52)$ & 0.39 & $1.8(1.44-2.24)$ & $<0,001$
\end{tabular}

\section{Discusión}

En la actualidad no existe una definición estandarizada de DCPO. Una diferencia importante entre DCPO y el trastorno neurocognitivo es el requisito de una preocupación cognitiva en este último y la evidencia del compromiso de la capacidad funcional. El consenso para la nomenclatura del cambio cognitivo asociado con la anestesia y la cirugía 2018, sugiere utilizar el término general de "trastornos neurocognitivos perioperatorios" para denominar al deterioro cognitivo identificado en el período preoperatorio o postoperatorio. Esto incluye el deterioro cognitivo diagnosticado antes de la operación, descrito como trastorno neurocognitivo (TNC) y cualquier forma de evento agudo (delirio postoperatorio) y deterioro cognitivo diagnosticado hasta 30 días después del procedimiento (recuperación neurocognitiva tardía). El término se convierte en trastorno neurocognitivo postoperatorio leve o mayor hasta después de 12 meses si los signos y síntomas de la disfunción cognitiva persisten más allá de lo esperado para la recuperación física, fisiológica y emocional de la cirugía (13). También por consenso se sugiere que estos trastornos no deben diferenciarse de los trastornos neurocognitivos en la población general y, por lo tanto, deben alinearse con el diagnóstico clínico y de acuerdo a los criterios de trastornos neurocognitivos utilizados en el Manual diagnóstico y estadístico para los trastornos mentales, Quinta edición (DSM-5).

Los criterios objetivos para los TNC son una disminución de 1 a 2 desviaciones estándar, generalmente contra un grupo normativo para leves y $>2$ desviaciones estándar. Para TNC mayores, se alinean con los criterios estándar de diagnóstico clínico, en déficit neurocognitivo leve o mayor (DSM-5).

El DCPO puede afectar a pacientes quirúrgicos de cualquier edad, pero se ha observado mayor frecuencia y mayor prolongación en pacientes de edad avanzada. En esta población se observa mayor riesgo de déficit cognitivo y aumento de la morbimortalidad en un $25 \%$ posterior a cirugías de alta complejidad (14). Se estima que así como aumenta el riesgo de cirugía en pacientes adultos mayores de igual manera aumente la probabilidad de desarrollar DCPO (15).

En los últimos años, el DCPO ha sido objeto de mayor revisión, y se han sugerido varios factores fisiopatológicos, incluyendo los procesos anestésicos, el proceso inflamatorio posquirúrgico (8), la predisposición genética en pacientes portadores del alelo E4 de la apolipoproteína E (APOE), polimorfismos que se han relacionado con alteración de la respuesta al trauma, deterioro cognitivo relacionado con la edad y delirium postoperatorio $(15,16)$. Por tanto, se determina un mecanismo multicausal con alteraciones de varios sistemas de neurotransmisores y del sistema colinérgico, presentándose disminución de neurotransmisores como acetilcolina, GABA y un aumento de dopamina, serotonina y cortisol (14).

En relación con la técnica anestésica, diversos estudios comparan el deterioro cognitivo entre anestesia general vs regional sugiriendo una asociación y mayor incidencia entre DCPO y anestesia general comparada con anestesia epidural $(17,18)$. Sin embargo, recientes estudios han concluido que no existe relación entre técnica anestésica general vs regional y la magnitud o aparición de DCPO (19).

Aunque la demencia y el déficit cognitivo no son causados por la cirugía, el cerebro es vulnerable en el período perioperatorio y es importante mitigar las consecuencias neurológicas indiscutibles de la cirugía mayor tales como delirium, DCPO temprano o accidente cerebro vascular (8). Uno de los estudios más relevantes realizados para evaluar el déficit cognitivo es el International Study of Postoperative Cognitive Dysfunction (ISPOCD), en el que se analizó a 1,218 pacientes mayores de 60 años con un procedimiento quirúrgico abdominal y torácico no cardiaco. Comparados con 321 controles, los principales resultados evidenciaron que al séptimo día del postoperatorio el $25.8 \%$ de 1.011 pacientes presentaron DCPO vs 3.4\% de 176 pacientes del grupo control. Posteriormente se hizo seguimiento a los tres meses, y el 9.9\% (20) presentó DCPO vs $2.8 \%$ de los controles. En diversos estudios se ha observado que la prevalencia es mayor del séptimo al décimo día de postoperatorio, y a su vez disminuye con el tiempo: 10\% a los tres meses, 5\% a los seis meses y $1 \%$ al año. En general la prevalencia de DCPO en pacientes mayores de 60 años es de $15-25 \%$, y que aproximadamente $10 \%$ la desarrollará a los tres meses posteriores a la cirugía (21). 
Con relación al delirium, la incidencia oscila entre 5.1\% y $52.5 \%$ en pacientes ancianos llevados a cirugía mayor, relacionándose con mayor riesgo la cirugía de cadera y aórtica; en neurocirugía es de $21.4 \%$. Con relación a los costos, el delirium genera aumentos en la estancia hospitalaria con gastos superiores 7,5 millones/día de hospitalización, con un costo de más de 4 billones de dólares con una población de adultos mayores hospitalizados superior a los 2,3 millones, sumado a los aumentos posteriores al egreso por rehabilitación, servicios ambulatorios e interconsultas $(22,23)$.

El MMSE ha alcanzado una amplia difusión debido a que es breve y sencillo tanto en su aplicación como en su puntuación y da información sobre el estado mental del paciente la cual es fácil de comunicar e interpretar. Como instrumento médico ha sido empleado en la detección y seguimiento del deterioro cognitivo, al igual que para evaluar la respuesta al tratamiento $(24,25)$. Adicionalmente se considera como un método para predecir el declive cognitivo en sujetos de edad avanzada (26); como herramienta de investigación, ha sido utilizado para detectar trastornos cognitivos en estudios epidemiológicos y para el seguimiento de cambios en el estado cognitivo en ensayos clínicos $(26,27)$. Posterior a su aplicación, se ha encontrado que en un individuo con deterioro leve del estado cognitivo los ítems que se afectan con mayor frecuencia son los relacionados con la memoria reciente y funciones de atención (recuerdo de tres objetos, memoria de la fecha y cálculo). En un estadio moderado los dominios comprometidos están relacionados con funciones de memoria a largo plazo como la orientación para el tiempo y el lugar. En un deterioro cognitivo severo los artículos perdidos incluyen la repetición de palabras, nombres de objetos simples y seguimiento de órdenes.

A pesar de considerar el tipo de cirugía y anestesia como desencadenantes del DCPO, se presentan estudios que lo refutan y proporcionan un enfoque diferente en cuanto a los factores asociados para el desenlace del DCPO. En particular se realizó un estudio donde se examinó la asociación entre la exposición a la cirugía y la cognición a largo plazo en una cohorte danesa de 8,503 pacientes de mediana edad y gemelos mayores. Se encontró una baja relevancia clínica en el DCPO con relación a la cirugía o anestesia. Mientras que la trayectoria cognitiva preoperatoria y la carga de la enfermedad coexistente fueron predictores más fuertes de la trayectoria cognitiva postoperatoria a largo plazo (28).

Los principales factores de riesgo involucrados para el desarrollo de DCPO son la edad avanzada, especialmente desde los 65 años. Millones de personas en el mundo son sometidas a cirugía bajo anestesia cada año y muchas de ellas son realizadas en población adulta mayor, quienes tienen una mayor tasa de cirugías en comparación con la población joven (29), una segunda cirugía, la duración de la anestesia, infecciones posoperatorias, complicaciones pulmonares, circulación extracorpórea $(30,31)$. La edad avanzada se asocia un tipo específico de enfermedades, especialmente las neurodegenerativas, las cuales tienen un componente genético determinado por la presencia del alelo E4 de la lipoproteína ApoE que está relacionada con la movilización y redistribución del colesterol y a su vez con la reparación, el mantenimiento y el crecimiento de membranas neuronales y mielina. Estas presentan una mayor incidencia después de los 80 años de edad debido a que el cerebro es más vulnerable por tener una menor densidad y plasticidad neuronal, además de más probabilidad de presentar lesiones y anormalidades cerebrales previas; también bajos niveles de neurotransmisores como la acetilcolina, dopamina y serotonina, y cambios que incrementan la incidencia de enfermedades neurodegenerativas como la enfermedad de Alzheimer (EA), la enfermedad de Parkinson (EP) y como otros tipos de demencia (32). La predisposición genética sumada a una edad avanzada es un factor de riesgo relevante para el desarrollo de DCPO (15).

Los anestésicos son importantes modificadores de la función cognitiva. Uno de los principales implicados es la ketamina y los agonistas GABA, que alteran la translocación de proteínas Bax en las membranas mitocondriales implicadas en la neuroapoptosis, lo que resulta en elevación en la permeabilidad de las membranas, activación de la caspasa-3 y de la cascada neuroapoptótica. Además, la ketamina y midazolam interfieren con la sinaptogénesis y la interacción celular. En relación con los anestésicos inhalados, el Isofluorano induce la activación de la caspasa-3 y apoptosis de una manera dependiente $(33,34)$. También disminuye la proliferación y aumenta la diferenciación neuronal, lo que conduce a disfunción cognitiva en recién nacidos y lesiones de la estructura del hipocampo durante el período crítico del desarrollo. El efecto de la anestesia sobre la función cognitiva depende de la farmacodinámica y cinética de los agentes particulares utilizados, cuanto menor sea la duración de la acción del agente anestésico, menor será la duración del deterioro cognitivo en el período postoperatorio a corto plazo.

Otros medicamentos utilizados durante el acto anestésico interfieren con la neurotransmisión colinérgica, lo que favorece el desarrollo de delirium y DCPO. Dentro de estos, el atracurio y su producto metabólico laudanósido 
que activan receptores colinérgicos muscarínicos. La morfina que antagoniza los receptores colinérgicos muscarínicos M1, M2 y M3; el fentanil un antagonista competitivo del receptor M3. El trauma quirúrgico y la respuesta neuroendocrina modifican la concentración de neurotransmisores (34).

Hasta la fecha, no se han encontrado pruebas definitivas para la hipótesis de que la anestesia en sí misma causa DCPO prolongado (35). En el momento no hay evidencia definitiva de que los fármacos anestésicos sean neurotóxicos. Con la técnica, diversos estudios previos comparan el deterioro cognitivo entre anestesia general vs regional sugiriendo una asociación y mayor incidencia entre DCPO y anestesia general comparada con anestesia epidural $(17,18)$. Aunque recientes estudios han concluido que no existe relación entre técnica anestésica general vs regional y la magnitud o aparición de DCPO (20).

Literatura reciente ha reportado el beneficio en la prevención del DCPO con el uso del BIS, observándose en pacientes mayores o igual de 60 años que se sometieron a cirugías mayores o no cardiacas y fueron seleccionados al azar para el uso de BIS. La anestesia fue guiada evitando que fuera profunda y así se la asoció a un mejor pronóstico y evitando la presencia de delirium durante la hospitalización, al igual que en los 3 meses postquirúrgicos (36). Jianxiong An et al., determinaron que TIVA guiada por el índice biespectral (BIS) que es el equipo de monitorización de la profundidad anestésica utilizado con mayor frecuencia, BIS permite manejar niveles más bajos anestésicos, lo cual disminuye la incidencia de DCPO en el postoperatorio inmediato. Mathew Chan et al., concluyeron que la anestesia guiada por BIS reduce la exposición anestésica y disminuye el riesgo de DCPO en los 3 meses posteriores a la cirugía, y por cada 1000 pacientes adultos mayores sometidos a cirugía mayor manejados con BIS 40-60 previene 23 casos de DCPO Y 83 casos de delirium (37).

\section{Conclusiones}

En la actualidad no existe una definición estandarizada de DCPO, ni se ha establecido alguna diferencia importante entre DCPO y el trastorno neurocognitivo. Los mecanismos que conducen al deterioro cognitivo después de la anestesia y la cirugía aún no están del todo claros. Todas las personas que atienden a pacientes intra y postoperatoriamente deben saber sobre el riesgo de DCPO después de la anestesia y la cirugía.
En cuanto a la incidencia de DCPO, se demostró que el temprano es significativamente menor comparándolo con lo reportado en la literatura internacional. Al comparar el DCPO tardío, el presente estudio muestra una incidencia mayor. Esto puede deberse a mayores eventos intraoperatorios presentados. Se observó que el $43.75 \%$ de los pacientes con hipotensión presentó DCPO. Los resultados muestran que factores como la edad, escolaridad y técnica anestésica influyen en el desarrollo de DCPO. El analfabetismo puede estar asociado con otras características que también están relacionadas como factor de riesgo como la edad avanzada, pobre estado cognitivo y un pobre estado funcional. Además, este comportamiento asociado a la edad se equipara a lo observado por Canet et al., quienes evaluaron el DCPO, en pacientes sometidos a cirugías menores. En el subgrupo de pacientes mayores a 75 años el DCPO fue del 18\% (38).

No se logró demostrar que las enfermedades crónicas, así como el tiempo quirúrgico influyeron en una mayor incidencia de DCPO, lo cual se correlaciona con otros estudios. Esto podría estar relacionado con que los pacientes al ser programados de manera ambulatoria tenían un adecuado control de sus patologías de base.

Los pacientes de edad avanzada deben estar seguros de que la cirugía y la anestesia probablemente no estarán implicadas en causar deterioro cognitivo persistente o demencia incidental. Aunque es importante que los investigadores centren su atención en las estrategias para prevenir el delirio postoperatorio, DCPO temprano y accidente cerebro-vascular peri operatorio.

Como limitaciones para la realización de este estudio se tiene en cuenta que únicamente se investigó en una institución de salud, además de que se tomaron pacientes mayores de 55 años, dado que no fue posible recolectar la muestra por la limitación en la cantidad de pacientes mayores de 65 años. Se declara que no existen conflictos de interés.

\section{Recursos}

El presente estudio se realizó con fondos propios del autor e investigador. Para su financiación no se contó con ningún patrocinio.

La papelería fue aportada por el estudio, al igual que elementos para diligenciar los formatos, como bolígrafos, tablas y demás elementos. 
El tiempo utilizado para la captación de pacientes, toma de datos, seguimiento y análisis fue determinado por el programa de posgrado.

\section{REFERENCIAS}

1. Sieber FE, Barnett SR. Preventing postoperative complications in the elderly. Anesthesiology Clinics. 2011;29(1):83-97. Doi: https://doi.org/10.1016/j. anclin.2010.11.011

2. Inouye SK, Westendorp RG, Saczynski JS. Delirium in elderly people. Lancet. 2014;383:911-22. Doi:https:// doi.org/10.1016/S0140-6736(13)60688-1

3. Agnoletti V, Ansaloni L, Catena F, Chattat R, De Cataldis A, Di Nino G, et al. Postoperative Delirium after elective and emergency surgery: analysis and checking of risk factors. A studyprotocol. BMC Surgery. 2005;5:12-18. Doi: https://doi. org/10.1186/1471-2482-5-12

4. Johnson T, Monk T, Rasmussen LS, Abildstrom $\mathrm{H}$, Houx $\mathrm{P}$, Korttila $\mathrm{K}$, et al. Postoperative cognitive dysfunction in middle-aged patients. Anesthesiology. 2002; 96:1351-7. Doi: https://doi. org/10.1097/00000542-200206000-00014

5. Newman MF, Grocott HP, Mathew JP, White WD, Landolfo K, Reves JG, et al. Neurologic Outcome Research Group and the Cardiothoracic Anesthesia Research Endeavors (CARE) Investigators of the Duke Heart Center: Report of the substudy assessing the impact of neurocognitive function on quality of life 5 years after cardiac surgery. Stroke. 2001; 32:287481. Doi: https://doi.org/10.1161/hs1201.099803

6. Phillips-Bute B, Mathew JP, Blumenthal JA, Grocott HP, Laskowitz DT, Jones RH, et al. Association of neurocognitive function and quality of life 1 year after coronary artery bypass graft (CABG) surgery. Psychosom Med. 2006;68:369-75. Doi: https://doi. org/10.1097/01.psy.0000221272.77984.e2

7. Monk TG, Weldon BC, Garvan CW, Dede DE, van der Aa MT, Heilman KM, et al. Predictors of cognitive dysfunction after major noncardiac surgery. Anesthesiology. 2008; 108:18-30. Doi: https://doi. org/10.1097/01.anes.0000296071.19434.1e

8. SteinmetzJ,ChristensenKB,LundT,LohseN, Rasmussen LS. Long-term consequences of postoperative cognitive dysfunction. Anesthesiology. 2009;110(3):548-55. Doi: 10.1097/ALN.0b013e318195b569

9. Guzmán V. Disfunción cognitiva postoperatoria en pacientes mayores de 50 años bajo anestesia general y regional. Estudio cohorte prospectivo. Biblioteca Virtual UIS.

10. Folstein MF, Folstein SE, McHugh PR. 'MiniMental State': a practical method for grading the cognitive state of patients for the clinician. J Psychiatr Res. 1975; 12:189-198. Doi: https://doi.org/10.1016/00223956(75)90026-6

11. Horton AM, Slone DG, Shapiro S. Neuropsychometric correlates of the Mini-Mental State Examination: preliminary data. Percept Mot Skills. 1987; 65:64-66. Doi: https://doi.org/10.2466/pms.1987.65.1.64

12. Anthony JC, LeResche L, Niaz U, Von Korff MR, Folstein MF. Limits of the 'Mini-Mental State' as a screening test for dementia and delirium among hospital patients. Psychol Med. 1982;12:397-408. Doi: https://doi.org/10.1017/S0033291700046730

13. Evered L, Silbert B, Knopman DS, Scott DA, DeKosky T, Rasmussen LS, et al. Recommendations for the Nomenclature of Cognitive Change Associated with Anaesthesia and Surgery-2018. Anesthesiology. 2018;129(5):872-879. Doi: https://doi.org/10.1097/ ALN.0000000000002334

14. Trzepacz PT, Van der Mast RC, Lindesay J, Rockwood K, MacDonald A. Pathophysiology of delirium. Delirium in old age [Internet]. Oxford University Press. 2002 [citado 19 de octubre de 2020];51-90. Recuperado a partir de: https://global. oup.com/academic/product/delirium-in-old-age 9780192632753? cc $=$ co\&lang $=$ en\&

15. Tsai T, Sands LP, Leung JM. An Update on Postoperative Cognitive Dysfunction. Advances in Anesthesia. 2010;28(1):269-284. Doi: https://doi. org/10.1016/j.aan.2010.09.003

16. Tsuang DW, Bird TD. Genetics of dementia. Medical Clinics of North American. 2002; 86:591-614. Doi: https://doi.org/10.1016/S0025-7125(02)00003-2

17. Hole A, Terjesen T, Brevik H. Epidural versus general anaesthesia for total hip arthroplasty in elderly patients. Acta Anaesthesiologica Scandinavica. 1980; 24:279287. Doi: https://doi.org/10.1111/j.1399-6576.1980. tb01549.x

18. Berggren D, Gustafson Y, Eriksson B, Bucht G, Hansson LI, Reiz S, et al. Postoperative confusion after anesthesia in elderly patients with femoral neck fractures. Anesthesia\&Analgesia Journal. 1987;66:497-504. Doi: https://doi. org/10.1213/00000539-198706000-00003

19. Williams-Russo P, Sharrock N, Mattis S, Szatrowski T, Charlson M. Cognitive effects after epidural vs general anesthesia in older adults. JAMA. 1995;274:44-50. Doi: https://doi.org/10.1001/ jama.1995.03530010058035

20. RasmussenLS,LarsenK,HouxP,SkovgaardLT,Hanning CD, Möller JT. ISPOCD group. The International Study of Postoperative Cognitive Dysfunction. The assessment of postoperative cognitive function. Acta Anaesthesiologica Scandinavica. 2001;45:275-89. Doi: https://doi.org/10.1034/j.1399-6576.2001.045003275.x 
21. Taylor D, Lewis S. Delirium. Journal of Neurology, Neurosurgery \& Psychiatry. 1993;56:742-751. Doi: https://doi.org/10.1136/jnnp.56.7.742

22. Carrillo-Esper R, Peña-Pérez CA, Gómez-Hernández K, Carrillo-Córdova LD, Villena-López EL. Disfunción cognitiva postoperatoria. RevInvestMed Sur (Mex) [Internet]. 2012 [citado 19 de octubre de 2016];19(3):163-169. Recuperado a partir de: https://www.medigraphic.com/cgi-bin/new/resumen. cgi? IDARTICULO $=41961$

23. Yoon-Sik O. Incidence and risk factors of acute postoperative delirium in geriatric neurosurgical patients. Journal of KoreanNeurosurgical Society. 2008;43:143-148. Doi: https://doi.org/10.3340/ jkns.2008.43.3.143

24. Folstein MF, Folstein SE, McHugh PR. 'MiniMental State a practical method for grading the cognitive state of patients for the clinician. J Psychiatr Res. 1975;12:189-198. Doi: https://doi.org/10.1016/00223956(75)90026-6

25. Horton AM, Slone DG, Shapiro S. Neuropsychometric correlates of the MiniMental State Examination: preliminary data. Percept Mot Skills. 1987;65:64-66. Doi: https://doi.org/10.2466/pms.1987.65.1.64

26. Gauthier S, Bouchard R, Lamontagne A, Bailey P, Bergman H, Ratner J, et al. Tetrahydroaminoacridinelecithin combination treatment in patients with intermediate-stage Alzheimer's disease: results of a Canadian doubleblind, crossover, multicenter study. N Engl J Med. 1990;322:1272-1276. Doi: https://doi. org/10.1056/NEJM199005033221804

27. Rosselli D, Ardila A, Pradilla G, Morillo L, et al. The mini-mental state examination as a diagnostic selection test for dementia: a colombian population study. Rev Neurol 2000; 30 (5): 428-432. https://doi. org/10.33588/rn.3005.99125

28. Dokkedal U, Hansen TG, Rasmussen LS, MengelFrom J, Christensen K. Cognitive functioning after surgery in middle-aged and elderly Danish twins. Anesthesiology. 2016;124:312-21. Doi: https://doi. org/10.1097/ALN.0000000000000957

29. Lobo F. Anestesia, demencias y enfermedad de Alzheimer: ¿coincidencia o certeza? Rev Argent Anestesiol [Internet] . 2012 [Citado 19 de octubre de 2020];70:91-6. Recuperado a partir de: https://www.anestesia.org.ar/search/articulos completos/1/1/1442/c.pdf

30. Deiner S, Silverstein JH. Postoperative delirium and cognitive dysfunction. British Journal of Anaesthesia. 2009;103(Suppl.1):i41-i46. Doi: $\quad$ https://doi. org/10.1093/bja/aep291

31. Avidan MS, Evers AS. Review of clinical evidence for persistent cognitive decline or incident dementia attributable to surgery or general anesthesia. Journal of Alzheimer's Disease. 2011;24(2):201-16. Doi: https://doi.org/10.3233/JAD-2011-101680

32. Rundshagen I. Postoperative cognitive dysfunction. Deutsches Arzteblatt international. 2014;111(8):119125. Doi: https://doi.org/10.3238/arztebl.2014.0119

33. Culley DJ, Baxter MG, Crosby CA, Yukhananov R, Crosby G. Impaired acquisition of spatial memory 2 weeks after isoflurane and isoflurane- nitrous oxide anesthesia in aged rats. Anesthesia\&Analgesia Journal. 2004;99:1393-1397. Doi: https://doi. org/10.1213/01.ANE.0000135408.14319.CC

34. Shors TJ, Miesegaes G, Beylin A, Zhao M, Rydel T, Gould E. Neurogenesis in the adult is involved in the formation of trace memories. Nature. 2001;410:372376. Doi: https://doi.org/10.1038/35066584

35. Lira D, Mar-Meza M, Montesinos R, Herrera-Pérez E, Cuenca J, Castro-Suárez S, et al. Una complicación quirúrgica escasamente sospechada: la disfunción cognitiva postoperatoria. Rev Neuropsiquiatria. 2018;81(2):113. Doi: https://doi.org/10.20453/rnp. v81i2.3339

36. An J, Fang Q, Huang C, Qian X, Fan T, Lin Y, et al. Deeper Total Intravenous Anesthesia Reduced the Incidence of Early Postoperative Cognitive Dysfunction After Microvascular Decompression for Facial Spasm. Journal of Neurosurgical Anesthesiology. 2011;23(1):12-7. Doi: https://doi. org/10.1097/ANA.0b013e3181f59db4

37. Chan M, Cheng P, Lee T, Gin T. BIS-guidedAnesthesia Decreases Postoperative Delirium and Cognitive Decline. Journal of Neurosurgical Anesthesiology. 2013;25(1):33-42. Doi: https://doi.org/10.1097/ ANA.0b013e3182712fba

38. Canet J, Raeder J, Rasmussen LS, Enlund M, Kuipers HM, Hanning CD, et al. Cognitive dysfunction after minor surgery in the elderly. Acta anaestheesiol Scand 2003;47:1204-1210. Doi: https://doi.org/10.1046/ j.1399-6576.2003.00238.x 